\title{
Anxiety and Social Introversion : comparing patients of schizophrenia and depression
}

\author{
Dasyl D'Cunha ${ }^{1}$, Sheba Kumar ${ }^{2}$ \\ ${ }^{1}$ Post graduate student, Department of Psychology, LJNJ College, Mumbai. \\ ${ }^{2} \mathrm{Head}$, Department of Psychology, LJNJ College, Mumbai. \\ E-mail-dasyldcunha@yahoo.co.in
}

\begin{abstract}
Background: Anxiety and social introversion are common symptoms that are seen in both depression and schizophrenia. They may be a part of the disorder itself or may be part of an independent anxiety condition that co-exists with the disorders. The current study was aimed at assessing the levels of anxiety and social introversion in patients of schizophrenia and depression and comparing the levels in these disorders.

Methods: 30 patients with depression and 30 patients with schizophrenia were subjects for the study. They were assessed as per the inclusion criteria and were administered the Beck Anxiety Inventory (BAI) and Social Introversion Scale. The scores were analysed statistically and presented.

Results: Both groups were well matched socio-demographically while age of onset was lower in patients with schizophrenia. Patients with schizophrenia had higher levels of anxiety than patients with depression $(p=0.048)$ while patients with depression had far higher scores on social introversion when compared to patients with schizophrenia $(\mathrm{p}<0.0001)$.

Conclusions: Social introversion and anxiety are important symptoms in patients with schizophrenia and depression and need further clinical understanding and exploration via future research.
\end{abstract}

Key words: anxiety, social introversion, schizophrenia, depression, Beck Anxiety Inventory, Social Introversion Scale.

(Paper received $-22^{\text {nd }}$ April 2016, Peer review completed $-28^{\text {th }}$ June 2016, Accepted $-20^{\text {th }}$ July 2016)

\section{INTRODUCTION}

Schizophrenia and depression are two of the commonest disorders seen in clinical practice. Anxiety and social introversion are common symptoms in both these disorders [1].

\section{Schizophrenia, Anxiety and Social Introversion}

Anxiety is frequently observed among patients with schizophrenia. Its presence can be conceptualized as a component of the presentation of schizophrenia itself, particularly during an acute psychotic episode and as a result of an underlying organic condition, a medication side effect, or a symptom of a comorbid anxiety disorder [2]. Higher anxiety ratings have been noted in patients with schizophrenia and are associated with less satisfaction with global quality of life, daily activities, family, health and social relationship, even when controlling for positive symptoms, negative symptoms [3].

Researchers have examined the interaction of specific anxiety subtypes and psychosis and concluded that anxiety disorders are common in patients with schizophrenia. The presence of such symptoms may influence the presence of core psychotic symptoms and quality Of life [4]. Other studies have reported high rates of social anxiety in clinical samples of patients with schizophrenia. Of note, half of the subjects with 
comorbid anxiety and psychosis reported that the anxiety disorder had predated the psychosis by several years. In addition, almost none of the patients had been diagnosed or treated for their anxiety disorder [5]. A study aimed to assess the prevalence of posttraumatic stress disorder (PTSD) after an acute psychotic episode in schizophrenic and delusional patients and to explore which psychotic symptoms and aspects of treatment were associated with traumatization and to compare the extent of the traumatic impact of psychosis and involuntary hospitalization. These data suggest that positive and depressive/anxious symptomatology were associated with psychosis-related traumatic symptoms [6].

Longitudinal analysis of teachers' comments in school records of schizophrenics showed progressive deviance in their childhood social behavior and sharp differences between the sexes. Pre-schizophrenic girls were primarily introverted and pre-schizophrenic boys mainly disagreeable [7]. In a prospective epidemiological study, 28 schizophrenics were assessed with a multi-dimensional personality test on average 2 years prior to the onset of their illness. The self-rating results show that schizophrenics are introverted in their premorbid personality and as compared to control groups; it is assumed that this personality trait will be understood as "schizoid" in non-self-rating. Surprisingly, schizophrenics premorbidly have a normal neuroticism score, i.e. they are not more irritable, more aggressive, more depressive or emotionally unstable than controls [8].

Research has indicated that reduced perceptual constancy is often reported in schizophrenia. Research has suggested the paranoid non-paranoid distinction reflects extraversion-introversion in schizophrenia and there are a number of studies of non-schizophrenics indicating that reduced constancy is common with introverts. They consider the increased susceptibility in schizophrenia to be a peculiarity of the illness [9]. In a study that compared personality trait profiles in patients with schizophrenia and healthy controls, male outpatients with schizophrenia and male non-psychiatric community samples completed the NEO-5 personality questionnaire. The overall personality profile of clinically stable patients with schizophrenia differed significantly from that of a community sample. On individual scales, patients scored significantly higher on neuroticism and significantly lower on conscientiousness [10].

\section{Depression, Anxiety and Social Introversion}

A study personality and depression was conducted to clarify the relationship between personality and depression. Thirty-one female patients with primary non-bipolar major depressive disorder were assessed diagnostically using the Schedule for Affective Disorders and Schizophrenia and completed a battery of standard self-report personality inventories when they were completely symptom free. Their personality scale scores were compared with those of female relatives who had recovered from the same type of disorder, those of female relatives with no history of psychiatric illness, and published scale norms. Compared with the normal population, both groups of recovered depressives were introverted, submissive, and passive, with increased interpersonal dependency but normal emotional strength [11].

Researchers have studied the relationship of neuroticism and extraversion to symptoms of anxiety and depression in the general population where In multiple regression models, even after adjusting for gender, age, and education, BDI scores were significantly associated with neuroticism, extraversion, and age, whereas BAI scores were associated only with neuroticism. Neuroticism is strongly associated with depressive and anxiety symptoms, and introversion is moderately associated with depressive symptoms in the urban general population [12].

Personality and psychopathology have been studied with an application of the Five-Factor Model and the relationship of the five-factor model of personality to psychopathology focusing in particular on Axis II personality disorders and depression have been researched. The five factors provide a particularly compelling model for interpreting the Axis II personality disorders as maladaptive variants of normal personality traits. Considerable attention was given to neuroticism and extraversion and in particular on the difficulty in distinguishing between the various ways in which personality can relate to depression, either as a predisposition to, a complication of, a pathoplastic effect upon, or a spectrum variant of the mental disorder [13].

The present study was conducted to assess and compare the levels of anxiety and social introversion in patients with depression and schizophrenia. It is hypothesized that schizophrenic patients have higher 
levels of anxiety compared to patients with depression. We are propose that depressed patients are more socially introverted than patients with schizophrenia

\section{METHODOLOGY}

30 consecutive patients with schizophrenia and depression visiting the psychiatric outpatient department made up the sample of the study (total 60). The patients were interviewed and surveyed at mental institutions and a local clinic. The patients were all having paranoid schizophrenia or in remission while others were mainly having major depressive disorder.

The design implemented was a random group design, having 2 independent variables and 2 dependent variables. Anxiety levels were compared between patients with schizophrenia and depression using the Beck Anxiety Inventory (BAI). Social Introversion was compared using the Introversion Scale.

All participants were diagnosed with schizophrenia and depression through psychometric assessment and clinical evaluation and met DSM-IVTR [14] criteria for the same. All participants had a minimum of $8^{\text {th }}$ grade education qualification.

Inclusion Criteria for patients in the study -

1. Age 20-50 years.

2. Diagnosis of depression and schizophrenia as per DSM-IVTR criteria and no other disorders.

3. The patients must not be having a diagnosis of mental retardation or personality disorder.

4. The patients must have good social support and must be staying with family members.

5. Absence of substance abuse except Nicotine dependence.

6. Absence of major medical and surgical illness that may affect study findings.

The participants were administered the following scales -

1. Beck's Anxiety Inventory (BAI): The Beck Anxiety Inventory is a well accepted self-report measure of anxiety in adults and adolescents for use in both clinical and research settings. It is a 21-item multiple-choice self-report inventory that measures the severity of an anxiety in adults and adolescents. Because the items in the BAI describe the emotional, physiological, and cognitive symptoms of anxiety but not depression, it can discriminate anxiety from depression. Although the age range for the measure is from 17 to 80 , it has been used in peer-reviewed studies with younger adolescents aged 12 and older. The BAI requires only a basic reading level, can be used with individuals who have intellectual disabilities, and can be completed in $5-10$ minutes using the pre-printed paper form and a pencil [15]. Respondents are asked to report the extent to which they have been bothered by each of the 21 symptoms in the week preceding (including the day of) their completion of the BAI. There is a lickert type scale from 0 to 3 . The values for each item are summed yielding an overall or total score for all 21 symptoms that can range between 0 and 63 points. A total score of 0 - 7 is interpreted as a "Minimal" level of anxiety; 8 - 15 as "Mild"; 16 - 25 as "Moderate", and; 26 - 63 as "Severe".

2. Introversion scale: This introversion scale was developed by McCroskey to be distinct from measures of communication apprehension [16]. Items are drawn from the work of Eysenck, with items which referenced communication excluded. This permits the measurement of introversion without the contamination of communication apprehension items and allows the examination of both introversion and communication apprehension as predictors of communication behaviors independently of each other. Items to measure neuroticism are used as filler items and are not scored with the introversion items. There are 18 items on a lickert type scale. Individuals scoring above 48 are highly introverted; those scoring below 24 have low introversion (are extraverted). Those scoring between 24 and 48 are in the moderate range.

Beck's Anxiety Inventory (BAI) and Introversion Scale were distributed to both groups of subjects. Each patient was administered the tests individually and instructions were provided at the time of test 
administration. They were assisted through the course of the test completion and were thanked for their participation at the end of the second test administration.

The BAI was distributed first and then a break of 20 minutes was given, following which the Introversion Scale was administered. After each of the inventories, the patients were asked their opinion about the inventories and whether they found the statements easy to relate to.

\section{STATISTICAL ANALYSIS}

Unpaired t test was used in the assessment of differences on the scores between scales across the groups of patients. All the statistical analysis was done using computerized Graph Pad statistical software.

\section{RESULTS}

Both groups of patients were well matched when compared from a socio-demographic perspective. The mean age of patients with schizophrenia was $33.7 \pm 6.9$ years and the mean age of the depression group was $34.2 \pm 7.2$ years. All patients were married and staying with their families. All patients belonged to lower socio-economic strata. Age of onset of illness on an average was lower in the schizophrenia group at $22.7 \pm 3.6$ years while the depressed group was $26.7 \pm 5.5$ years. Results of the study revealed that patients with schizophrenia had a highs score on anxiety when compared to patients with depression. This was statistically significant as well. Social introversion scores on the other hand were higher in patients with depression ( $p<0.05$ in both cases) [Table 1].

Table 1 - Comparison of scores on various scales across both the groups

\begin{tabular}{|c|c|c|c|}
\hline Parameter & $\begin{array}{l}\text { Schizophrenia Group } \\
\qquad(\mathrm{n}=30)\end{array}$ & $\begin{array}{l}\text { Depression group } \\
(\mathrm{n}=30)\end{array}$ & Statistical Analysis \\
\hline \multicolumn{4}{|c|}{ Mean \pm SD } \\
\hline Beck Anxiety Inventory & $46.33 \pm 6.71$ & $43.53 \pm 3.66$ & $\begin{array}{l}t=2.0065 \\
p=0.04^{*}\end{array}$ \\
\hline $\begin{array}{c}\text { Social Introversion } \\
\text { Scale }\end{array}$ & $44.07 \pm 7.02$ & $51.83 \pm 2.99$ & $\begin{aligned} t & =5.5706 \\
p & <0.0001^{*}\end{aligned}$ \\
\hline
\end{tabular}

*significant $(\mathrm{p}<0.05)$. Analysis done using Unpaired t test

\section{DISCUSSION}

One of the two most evident findings revealed higher anxiety levels among individuals with schizophrenia when compared to those with depression. This finding was in line with a study conducted which revealed highest levels of anxiety among schizophrenic inpatients. These self-ratings of anxiety symptoms were significantly elevated across three psychoses, revealing highest among those diagnosed with schizophrenia [17]. Similar findings were reported by researchers who concluded from their study that anxiety was one of the principal symptoms among those suffering from schizophrenia, social anxiety in particular [18]. It has been observed that schizophrenia patients with prominent negative symptoms must be checked for anxious and depressive tendencies. They found that the patients exhibited significantly higher tendencies of anxiety and significantly lower tendencies of depression [19].

Authors investigated the relationship between social anxiety and positive and negative symptomatology in schizophrenia and found that Positive symptoms were related to fear in a number of self-report domains (i.e., social and agoraphobic). Negative symptoms on the other hand were related to global observational ratings of anxiety during the role play as well as specific behaviours associated with self-reported social anxiety (i.e., speech rate and fluency) [20].

The second resulting finding of this research revealed higher social introversion among individuals with depression when compared to those with schizophrenia. These results were not in line with research that has concluded that psychotic individuals are far more socially introverted than were depressive individuals [21]. Recent studies however, have revealed the contrary with depressive individuals increasingly preferring seclusion and becoming recluses [22]. Research has shown that associations between family 
socialization and depression along with concepts of self-efficacy and self-esteem are affected in these patients [23].

There are some limitations to the findings of the current paper. Firstly, participants of the study were not of one particular subtype of schizophrenia and this was not considered in the analysis. Secondly, individuals with major depression were involved in the study but the duration of their illness was not taken into consideration. Individuals with chronic depression as well as recently diagnosed as depressed were part of the study. Various illness parameters were not considered for both diagnoses. This paper however also posits that anxiety and social introversion are important variables in patients with schizophrenia and depression and need further understanding and research.

\section{REFERENCES}

1. Collins PY, Patel V, Joestl SS, March D, Insel TR, Daar AS, Bordin IA, Costello EJ, Durkin M, Fairburn C, Glass RI. Grand challenges in global mental health. Nature 2011;475(7354):27-30.

2. Cosoff SJ, Julian Hafner R. The prevalence of comorbid anxiety in schizophrenia, schizoaffective disorder and bipolar disorder. Aust NZ J Psychiatry 1998;32(1):67-72.

3. Pallanti S, Quercioli L, Hollander E. Social anxiety in outpatients with schizophrenia: a relevant cause of disability. Am J Psychiatry 2004;161(1):53-8.

4. Huppert JD, Smith TE. Anxiety and schizophrenia: the interaction of subtypes of anxiety and psychotic symptoms. CNS spectrums 2005;10(09):721-31.

5. Tibbo P, Swainson J, Chue P, LeMelledo JM. Prevalence and relationship to delusions and hallucinations of anxiety disorders in schizophrenia. Depress Anxiety 2003;17(2):65-72.

6. Scheller-Gilkey G, Moynes K, Cooper I, Kant C, Miller AH. Early life stress and PTSD symptoms in patients with comorbid schizophrenia and substance abuse. Schizophr Res 2004;69(2):167-74.

7. Camisa KM, Bockbrader MA, Lysaker P, Rae LL, Brenner CA, O'Donnell BF. Personality traits in schizophrenia and related personality disorders. Psychiatr Res 2005;133(1):23-33.

8. Lenzenweger MF. Schizotypy an organizing framework for schizophrenia research. Curr Dir Psychol Sci 2006;15(4):162-6.

9. Thorup A, Petersen L, Jeppesen P, Øhlenschlæger J, Christensen T, Krarup G, Jørgensen P, Nordentoft M. Social network among young adults with first-episode schizophrenia spectrum disorders. Soc Psych Psychiatr Epidemiol 2006;41(10):761-70.

10. Hori H, Noguchi H, Hashimoto R, Nakabayashi T, Saitoh O, Murray RM, Okabe S, Kunugi H. Personality in schizophrenia assessed with the Temperament and Character Inventory (TCI). Psychiatr Res 2008;160(2):175-83.

11. Selfhout MH, Branje SJ, Delsing M, ter Bogt TF, Meeus WH. Different types of Internet use, depression, and social anxiety: The role of perceived friendship quality. J Adolescence 2009 Aug 31;32(4):819-33.

12. Bienvenu OJ, Samuels JF, Costa PT, Reti IM, Eaton WW, Nestadt G. Anxiety and depressive disorders and the five-factor model of personality: A higher-and lower-order personality trait investigation in a community sample. Depress Anxiety 2004;20(2):92-7.

13. Biondi M, Picardi A, Pasquini M, Gaetano P, Pancheri P. Dimensional psychopathology of depression: detection of an 'activation'dimension in unipolar depressed outpatients. J Affect Disord 2005;84(2):133-9.

14. American Psychiatric Association. Diagnostic and Statistical Manual for the Classification of Psychiatric Disorders $-4^{\text {th }}$ edition - text revised. American Psychiatric Publishing ; 2000.

15. Leyfer OT, Ruberg JL, Woodruff-Borden J. Examination of the utility of the Beck Anxiety Inventory and its factors as a screener for anxiety disorders. J Anxiety Disord 2006;20(4):444-58.

16. McCroskey JC, Richmond VP, Heisel AD, Hayhurst JL. Eysenck's Big Three and communication traits: Communication traits as manifestations of temperament. Commun Res Reports 2004;21(4):404-10.

17. Achim AM, Maziade M, Raymond É, Olivier D, Mérette C, Roy MA. How prevalent are anxiety disorders in schizophrenia? A meta-analysis and critical review on a significant association. Schizophr Bull 2011;37(4):811-21.

18. Lysaker PH, Salyers MP. Anxiety symptoms in schizophrenia spectrum disorders: associations with social function, positive and negative symptoms, hope and trauma history. Acta Psychiatr Scand 2007;116(4):2908.

19. Braga RJ, Petrides G, Figueira I. Anxiety disorders in schizophrenia. Compr Psychiatry 2004;45(6):460-8.

20. Blanchard JJ, Cohen AS. The structure of negative symptoms within schizophrenia: implications for assessment. Schizophr Bull 2006;32(2):238-45. 
21. Karakurum B, Soylu Ö, Karatas M, Giray S, Tan M, Arlier Z, Benli S. Personality, depression, and anxiety as risk factors for chronic migraine. Int J Neurosci 2004;114(11):1391-9.

22. Dunkley DM, Sanislow CA, Grilo CM, McGlashan TH. Perfectionism and depressive symptoms 3 years later: Negative social interactions, avoidant coping, and perceived social support as mediators. Compr Psychiatry 2006;47(2):106-15.

23. Aluja A, Blanch A. Depressive mood and social maladjustment: Differential effects on academic achievement. Eur J Psychol Educn 2004;19(2):121-31.

Acknowledgements - Nil

Source of Funding - Nil

Conflict of Interest - Nil 